\title{
Hacia un nuevo modelo de ayudas al estudio y financiación universitaria
}

\author{
Manuel Valdés Fernández \\ Universidad Complutense de Madrid
}

\section{Resumen}

La composición social del estudiantado universitario español muestra una acusada infrarrepresentación de las clases bajas que la política de becas no ha logrado revertir. Menos aún será capaz de hacerlo tras la reforma del sistema de ayudas al estudio implementada a partir del curso 2012-2013 y mientras se mantenga una estructura lineal de precios públicos en la Universidad. Se antoja necesaria una nueva reforma que adapte el sistema de becas a las clases sociales cuyas decisiones educativas y rendimiento universitario se ven auténticamente condicionados por motivos económicos. Ello, junto a una nueva estructura de precios basada en la disponibilidad económica del estudiante y su familia, puede lograr una mayor participación de las clases bajas en la Universidad.

\section{Qué es un sistema de becas}

Los conceptos de beca y becario son términos enormemente connotados y polisémicos, refiriendo en el discurso público muy distintas realidades que trascienden la que aquí se pretende referir. Conviene siempre, por tanto, iniciar cualquier alocución sobre el sistema de becas con una definición clara de las ideas fundamentales que lo atañen. De acuerdo con el Real Decreto 1721/2007, por el que se estableció el régimen de becas y ayudas al estudio personalizadas, el sistema queda constituido por todas aquellas cantidades económicas concedidas a fin de matricularse o permanecer en el sistema reglado de enseñanza y distribuidas atendiendo criterios económicos y de rendimiento. Y becario será la condición de aquel que recibe una de estas ayudas.

Dicho sistema de becas tiene por objetivo, antes que cualquier otra cosa, compensar las desigualdades económicas entre los estudiantes, a fin de mejorar los niveles de eficiencia y equidad del sistema de enseñanza (Mediavilla, 2010). Nótese que, cuando el nivel de renta del hogar provoca la no culminación de cualquier nivel de estudios o su culminación con un 
aprovechamiento por debajo del potencial, se presentan situaciones en que, primero, la inversión de recursos realizada por el sistema educativo desaprovecha el potencial del estudiante, lo que es claramente un gasto ineficiente, $\mathrm{y}$, segundo, que afectan especialmente a aquellos hogares en que la disponibilidad económica es menor, contraviniendo así el principio de justicia social que debe regir el sistema de enseñanza.

No obstante, debe tenerse muy presente que el capital económico no es la única característica del origen social que condiciona el itinerario formativo de los estudiantes; esto es, que la disponibilidad de recursos económicos no es el único factor que provoca sesgos en el desempeño de los estudiantes en el medio escolar. El capital cultural, en sus múltiples operacionalizaciones y definiciones, se ha demostrado un predictor igualmente relevante de esos itinerarios formativos. Distintas investigaciones han abundando en el efecto que el capital cultural del hogar tiene tanto sobre el rendimiento académico del estudiante (Hernández \& González, 2011; Scandurra, 2013; Andersen, \& Nordli, 2012) como sobre el proceso de toma de decisión durante las transiciones entre niveles del sistema de enseñanza (Hillmert \& Jacob, 2003; Bodovski, 2010; Ustrell, 2012). Téngase en cuenta, además, que la mayor parte de las ayudas del sistema de becas se concentran en la educación postobligatoria, momento para el cual el origen social ha ejercido ya buena parte de su efecto sobre el itinerario formativo de los estudiantes (Río Ruiz, 2014).

Así pues, la capacidad de cualquier sistema de becas para corregir las desigualdades de partida es siempre limitada, al haber variables relevantes sobre las que no actúa. Olvidar esa realidad lleva a exigir del sistema de becas resultados que no es capaz de producir. Pero eso no cambia la inequívoca vocación compensatoria del sistema, que queda garantizada a través de un criterio de reparto construido a partir de la necesidad económica de los solicitantes.

Sí puede decirse, en cambio, que a través del sistema de becas debiera asegurarse que nadie abandona o deja de matricularse en cualquier nivel del sistema educativo debido a razones económicas. En otras palabras, que los costes directos (costes asociados a la matriculación o permanencia en el sistema de enseñanza), indirectos (costes derivados del estudio como los gastos de transporte hasta el centro de enseñanza) y de oportunidad (las retribuciones económicas que dejan de obtenerse por la decisión de retrasar la incorporación al mercado de trabajo) ocasionados por el estudio no condicionan las decisiones educativas de los estudiantes o su desempeño una vez adoptada una decisión. 


\section{Cómo afecta el sistema de becas al rendimiento y decisiones de los estudiantes}

Efectivamente, el sistema de becas tiene por objetivo evitar que los estudiantes tomen decisiones relativas a su matriculación o permanencia en cualquier nivel del sistema de enseñanza en función de la capacidad de su hogar para afrontar los costes directos, indirectos y de oportunidad asociados a los estudios postobligatorios. En clara relación con ello, también tiene por objetivo que el rendimiento académico de los estudiantes con rentas familiares más bajas no se vea mermado por esa menor disponibilidad de recursos. Diversas investigaciones llevadas a cabo tanto en la Educación Secundaria Superior (ESS) como en la educación universitaria han puesto de manifiesto el efecto que el sistema de becas puede tener sobre ambas cuestiones, revirtiendo situaciones que, de no corregirse, erosionarían la igualdad de oportunidades del sistema educativo.

Las investigaciones de Mediavilla $(2010,2013,2014)$ muestran el potencial del sistema de becas como dinamizador del rendimiento durante la ESS y potenciador de la matriculación en estudios posteriores. Utilizando datos de la Encuesta de Condiciones de Vida (ECV), implementa un diseño cuasi-experimental basado en la generación de un grupo de control (no becarios) a través de la técnica Propensity Score Matching con quienes comparar al grupo experimental (becarios). Mediavilla concluye que el hecho de disponer de una beca durante los estudios de Bachillerato y Grado Medio incrementa en más de un $20 \%$ las probabilidades de finalizar satisfactoriamente el nivel matriculado con respecto a sus compañeros no becarios. Asimismo, encuentra diferencias notables en el impacto de la beca en función del género y el origen social del becario. En el caso de las estudiantes becarias, el incremento de probabilidad de finalización de la ESS por el hecho de disponer de beca es tres veces el que caracteriza a los varones. Lo mismo observa para aquellos estudiantes cuyas madres presentan niveles formativos y laborales más bajos y que viven en hogares con una menor renta, aprovechando de nuevo más la ayuda que aquellos otros estudiantes también becarios que viven en hogares más acomodados (Mediavilla, 2013).

El estudio de Río y Jiménez (2015) sobre el programa de becas 6000, desarrollado en Andalucía y destinado a la ESS, apunta en la misma dirección. Si bien dichas ayudas requieren para su mantenimiento cumplir requisitos económicos notablemente más estrictos que el resto de becas, la investigación permite concluir que obtener una beca 6000 mejora tanto el rendimiento académico durante la ESS como las probabilidades de matriculación en estudios universitarios. Sin embargo, observan los autores que la probabilidad de matriculación en la propia ESS no se ve afectada por la existencia de dicho programa de becas, pues los estudiantes suelen cono- 
cer de su existencia una vez han comenzado los estudios de Bachillerato o Formación Profesional.

Resultados similares se desprenden del análisis del sistema de becas universitario. El estudio de Ruesga y Heredero (2010) mostró el efecto positivo que tanto la condición de becario como la cuantía de la beca tienen sobre el rendimiento en la Universidad. Para ello se valieron de una muestra de estudiantes universitarios españoles constituida en dos rondas en los años 2003 y 2006 a fin de analizar la composición social de ese estudiantado y los efectos del sistema de becas sobre sus decisiones educativas y rendimiento. Para afrontar el problema de la endogeneidad entre la probabilidad de obtener una beca y el desempeño académico, los autores se valieron de un modelo con variable instrumental. Observaron que la cuantía de la beca era un predictor del rendimiento mucho más importante que la renta del hogar y que cualquier otra transferencia pública, siendo que, por cada 100 euros de aumento en la cuantía de la beca, el porcentaje de asignaturas aprobadas en primera convocatoria (indicador de rendimiento académico utilizado) aumentaba 4,5 puntos porcentuales. Es más, cuando la variable introducida era la condición de becario en lugar de la cuantía, el hecho de recibir una beca incrementaba el rendimiento 8,1 puntos porcentuales. Se ponen así de manifiesto los potenciales efectos positivos del sistema de becas en el nivel universitario, no ya solo sobre la decisión de matricularse finalmente en tales estudios, sino a la hora de mejorar el rendimiento y evitar situaciones de abandono.

Los resultados de De Pablos y Gil Izquierdo (2008) apuntan en la misma dirección usando datos de la ECV del año 2004 para analizar la demanda de estudios universitarios de la población española a través de un modelo probit. Muestran cómo, cuanto mayor sea la proporción que la subvención media en una CCAA representa sobre la renta de un hogar, mayor es el deseo de acceder a la Universidad de los miembros de ese hogar. Encuentran también un efecto significativo, aunque de menor magnitud, del importe de las becas sobre dicha decisión.

Conclusiones algo disonantes se obtienen tras el estudio de las hoy extintas becas salario, ayudas de mayor cuantía vinculadas a estrictos criterios de renta. El estudio de Berlanga et al. (2010) utilizando los datos de registro de los estudiantes de la Universidad de Barcelona (UB) puso de manifiesto que la condición de becario en esta modalidad no suponía incrementos significativos de rendimiento respecto a los compañeros que no disponían de beca salario. Sí observaron, en cambio, un incremento en la probabilidad de acceder a la educación universitaria para aquellos estudiantes que habían finalizado un Ciclo Formativo de Grado Superior que no se extendía a la vía del Bachillerato y la Prueba de Acceso a la Universidad (PAU). Téngase en cuenta, no obstante, que para estos estudiantes receptores de la 
beca salario la cantidad dineraria recibida es condición sine qua non para su permanencia en la Universidad, de manera que ponen en marcha estrategias dirigidas a satisfacer los requisitos de mantenimiento de la beca que, en ocasiones, son contraproducentes a la hora de elevar la nota media de la carrera. Río (2014) ha estudiado las distintas estrategias desarrolladas por estudiantes universitarios receptores de ayudas públicas condicionadas al rendimiento, dando cuenta de la mayor importancia que tiene para dichos estudiantes no perder la beca por encima de obtener una calificación media más elevada. A la vez, no podrán poner en marcha otro tipo de estrategias, como la matriculación en un número menor de créditos, que sí están a disposición del estudiantado no becario (Berlanga et al., 2010). Pero no solo la condición de becario afecta al comportamiento de los estudiantes universitarios. Los estudiantes procedentes de clases sociales más bajas tienden a elegir carreras de menor dificultad, menor precio y, pensando en las ya extintas titulaciones de primer y segundo ciclo, de menor duración (Elias y Daza, 2014; Ruesga y Heredero, 2010). Y más allá de la probabilidad de matriculación y el logro académico, estudios cualitativos ponen en valor la capacidad del sistema de becas para la gestión del estrés emocional de los estudiantes de clases sociales más bajas. La decisión de prolongar su itinerario formativo matriculándose en estudios universitarios supone, para los hijos de familias de clase obrera, sentimientos de culpa y deuda por no insertarse profesionalmente y aportar económicamente al hogar (Río, 2014).

Finalmente, a nivel internacional son muchos los estudios dedicados a los llamados programas de transferencias de rentas condicionadas, que consisten en ayudas económicas que exigen determinado comportamiento por parte del estudiante receptor a fin de mantener la beca, como una asistencia mínima al aula o unos determinados resultados académicos al finalizar el año. De dichos estudios se desprende la capacidad de tales programas para prolongar la vida escolar de los estudiantes y retrasar su inserción en el mercado de trabajo, evitando repeticiones de curso y situaciones de abandono e incrementando el logro educativo en los niveles secundarios. Se muestra, además, su capacidad para aumentar, en algunos casos de forma notable, la matriculación en estudios posteriores, si bien la mayor parte de estos efectos positivos ocurren en países en desarrollo y no tanto al ser implementados en países desarrollados (Slavin, 2010).

\section{El sistema universitario de ayudas al estudio en España}

A fin de que el sistema de becas sea capaz de producir los mencionados resultados en los niveles no obligatorios y, en último término, favorecer la equidad y eficiencia global del sistema de enseñanza, debe estar adecuadamente configurado y dotado de recursos. 
El diseño del sistema de becas español se ha ido modificando a lo largo de los años, si bien no es posible hablar propiamente de un sistema público de ayudas al estudio anterior al año 1983. Con la promulgación del RD 2298/1983 se asientan las bases de un sistema que, progresivamente, fue aumentando sus niveles dotación y cobertura, quedando configurado a partir de un criterio de reparto basado en la concurrencia competitiva entre los solicitantes. Dicho principio suponía que la satisfacción de las condiciones de la ayuda no significaba su inmediata adjudicación, sino que, en función de la disponibilidad presupuestaria, más o menos estudiantes se convertían en beneficiarios del sistema. De acuerdo con los datos de Mohamed (2015), el sistema creció hasta el año 1996, momento en que se inicia un periodo de atonía e incluso decrecimiento que se prolonga hasta el año 2004 .

El sistema de becas recibió un nuevo impulso con la promulgación de la Ley 24/2005 (LRIP), aboliéndose el principio de concurrencia competitiva y pasando a considerar la beca como un derecho de todo aquel estudiante que satisficiese un conjunto de requisitos de renta y rendimiento. La nueva forma del sistema quedó consolidada a través del RD 1721/2007, que profundizaba la descentralización competencial del modelo de ayudas al estudio permitiendo que las CCAA participasen en la distribución de las becas. Todo ello a la vez que el porcentaje de estudiantes beneficiarios, la cuantía media de las ayudas y el gasto en becas en relación al total de gasto educativo seguían aumentando (Mohamed, 2015).

El sistema fue sustancialmente modificado a través del RD 609/2013, reforma anticipada por el Real Decreto-ley 14/2012 que permitió su aplicación ya durante el curso académico 2012-2013. La beca quedaba ahora dividida en dos partes. Una primera parte fija, a la que el estudiante tenía derecho una vez satisfechos los criterios de renta y rendimiento fijados. Y una segunda parte variable con nuevos criterios de rendimiento cuya cuantía dependía de la nota media y renta del estudiante y, lo que es más importante, del número de estudiantes solicitantes, lo que en la práctica suponía recuperar el principio de concurrencia competitiva. La introducción de una cuantía variable provoca, del lado de la Administración, un mayor control del presupuesto final dedicado a becas, en tanto que, una vez repartidas las cuantías fijas, es el montante restante hasta la cantidad presupuestada el que se distribuye en la forma de cuantías variables. A ello se añade el endurecimiento de los criterios académicos exigidos para la solicitud de la beca, que ha provocado un descenso en el número de estudiantes universitarios becarios (Hernández Armenteros y Pérez Días, 2014).

Actualmente, y de acuerdo con los datos del Ministerio de Educación Cultura y Deporte para el curso académico 2013-2014, un total de 322.753 estudiantes son beneficiarios del sistema de becas, lo que supone una tasa 
de cobertura del $24,6 \%$ que aumenta al 38,4\% si se consideran únicamente los estudiantes de nuevo ingreso (MECD, 2016).

Comparada con nuestro entorno internacional, la cobertura del sistema de ayudas al estudio en España puede considerarse baja (Gráfico 1). Según los datos de la Conferencia de Rectores de las Universidades Españolas, la tasa de cobertura del curso 2014-2015 en España se situó en el 27\%, muy lejos de países como Dinamarca, Finlandia u Holanda (CRUE, 2016). No obstante, cualquier comparación de las tasas de cobertura entre distintos sistemas universitarios debe tomar en consideración el distinto modelo de financiación que les caracterice, la composición social del estudiantado, el formato de las ayudas (becas o prestamos) y su cuantía media.

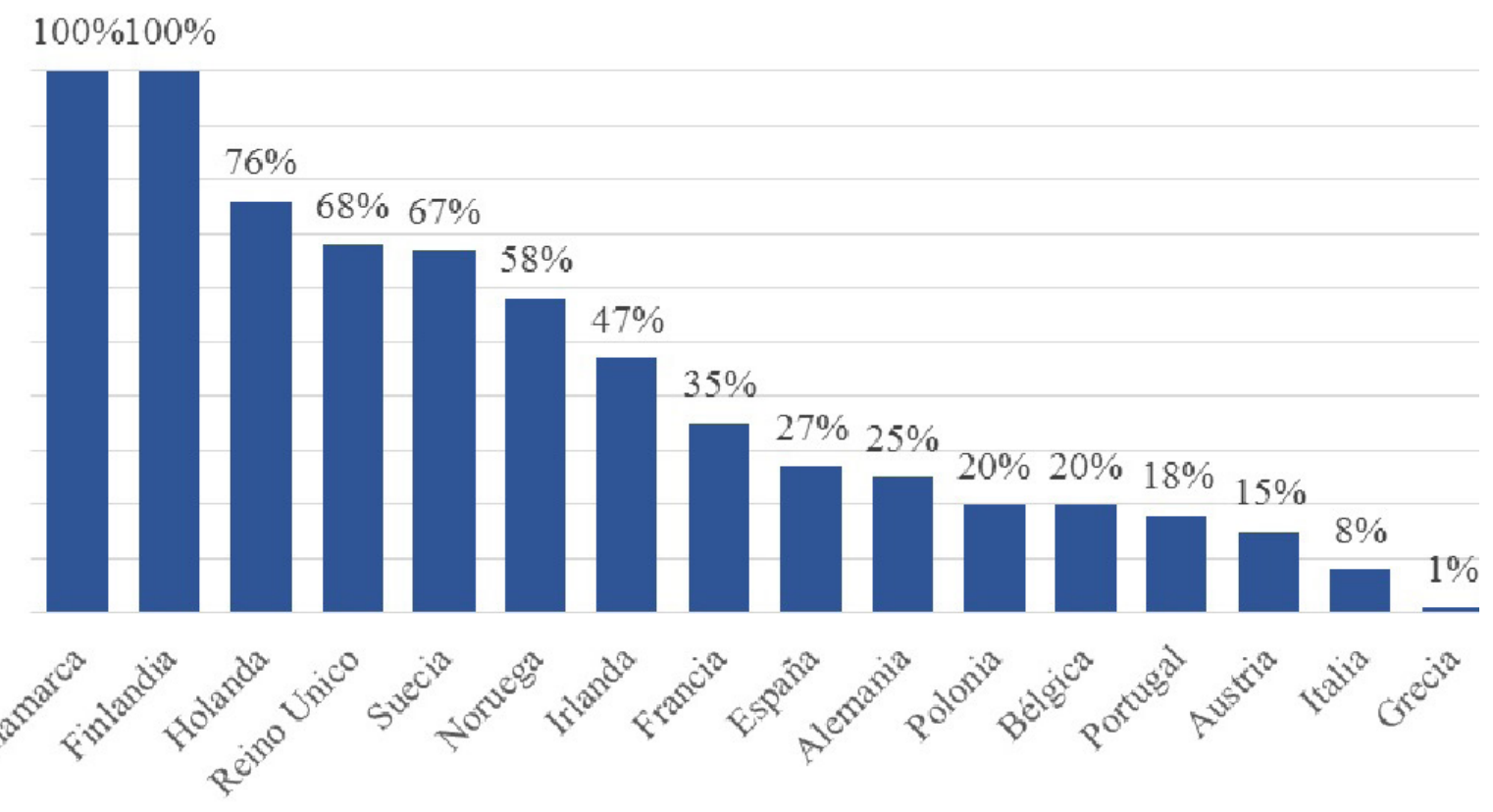

Gráfico 1. Porcentaje de estudiantes universitarios que reciben ayudas para el estudio en el curso 2014-2015. Fuente: CRUE, 2016.

Al respecto de la tasa de cobertura en nuestro país, Pérez Esparrells y Morales (2014) observan una correlación negativa significativa y fuerte entre los niveles de renta per cápita de las distintas Comunidades y el porcentaje de población universitaria receptor de una beca en cada una de ellas. Se puede afirmar, por tanto, que el reparto de las ayudas tiende a distribuirse siguiendo criterios de necesidad económica que se adaptan adecuadamente a las características de las distintas regiones de nuestro país; efecto que podría verse favorecido por una mayor descentralización del sistema.

En relación a la composición de las becas, de los 322.753 estudiantes beneficiarios en el curso académico 2013-2014, una quinta parte recibió únicamente la exención de tasas correspondientes a la matriculación, la denominada beca matrícula; y las otras cuatro quintas partes recibieron, 
además, la beca económica, cantidad dineraria compuesta por las partes fija y variable antes descritas. Todo ello supuso un gasto público en el sistema de becas de 803.244.716 euros (un 0,08\% del PIB del año 2013). Proporción que, como señalan distintos estudios, está por debajo de la media internacional, siendo que, además, entre los años 2000 y 2010 la diferencia entre España y la OCDE ha pasado de 0,16 puntos porcentuales a 0,20; es decir, nos hemos alejado de la media internacional (Mohamed, 2015).

\begin{tabular}{|l|l|l|l|l|l|}
\hline & $\begin{array}{l}\text { Estudiantes } \\
\text { solo con beca } \\
\text { matrícula }\end{array}$ & $\begin{array}{l}\text { Estudiantes } \\
\text { con beca } \\
\text { matrícula } \\
\text { y beca } \\
\text { económica }\end{array}$ & & \\
\hline & $\begin{array}{l}\text { Número de } \\
\text { beneficiarios }\end{array}$ & $\begin{array}{l}\text { Importe } \\
\text { concedido }\end{array}$ & $\begin{array}{l}\text { Número de } \\
\text { beneficiarios }\end{array}$ & $\begin{array}{l}\text { Importe } \\
\text { concedido }\end{array}$ & \\
\hline & & $\begin{array}{l}\text { Beca } \\
\text { matrícula }\end{array}$ & & $\begin{array}{l}\text { Beca } \\
\text { matrícula }\end{array}$ & $\begin{array}{l}\text { Beca } \\
\text { económica }\end{array}$ \\
\hline Grado & 57.514 & $775 €$ & 240.488 & $789 €$ & $2.093 €$ \\
\hline Máster & 572 & $1.228 €$ & 15.321 & $1.430 €$ & $2.004 €$ \\
\hline
\end{tabular}

Tabla 4. Distribución del presupuesto en ayudas al estudio durante el curso académico 20132014. Fuente: Elaboración propia a partir de MECD, 2016.

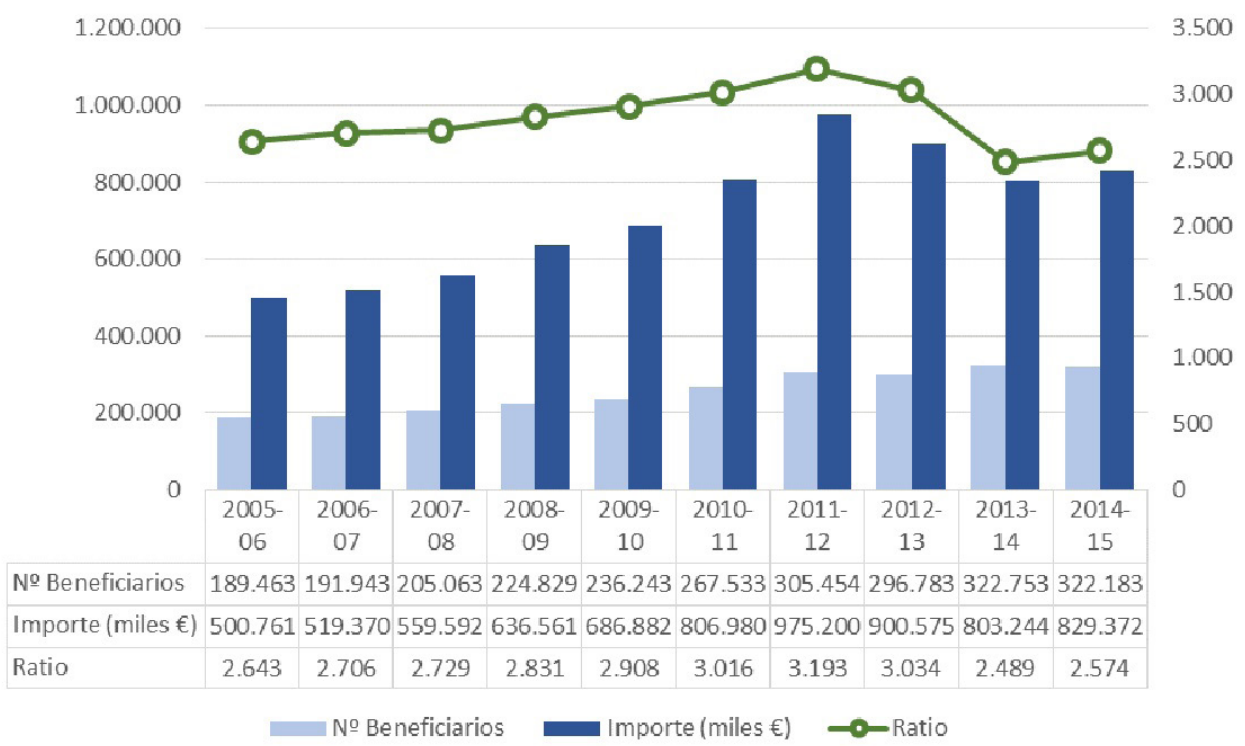

Gráfico 2. Número de Beneficiarios e importe dedicado al sistema de becas universitarias. Fuente: Elaboración propia a partir de MECD, 2007-2016. 
Del total de gasto en el sistema universitario de becas y el número de beneficiarios se obtiene la cuantía media de las ayudas, que para el curso 2013-2014 se situó en 2.489 euros. No obstante, dicha media presenta notables diferencias en función del nivel universitario matriculado y de si el estudiante recibió únicamente la beca matrícula o si recibió, además, la beca económica (Tabla 4). Cuando solo se obtiene la beca económica (19,3\% de los beneficiarios), la cuantía media asciende a 775 euros en el caso de los Grados y a 1.228 euros para los Máster. Al recibir ambas cuantías (80,7\% de los beneficiarios), las medias ascienden a 2.882 euros en Grado y a 3.434 euros en los Máster. Finalmente, tomando en consideración solo el dinero efectivamente desembolsado por la AGE hacia los universitarios y no las exenciones de tasas (dinero que deja de ingresar pero no abona), en el curso académico 2013-2014 se distribuyeron 534.061.256 euros entre un total de 255.809 beneficiarios. Es decir, la cuantía media efectivamente ingresada por aquellos estudiantes que, más allá de dejar de abonar las tasas reciben una cantidad dineraria, es de 2.088 euros de media.

A fin de apreciar la evolución del sistema de becas, y utilizado las publicaciones del MECD sobre el sistema universitario de los últimos diez años, hemos elaborado un gráfico (Gráfico 2) en el que se observa el número de beneficiarios del sistema de becas, el volumen total de recursos económicos dedicados a sufragar el sistema y la cuantía media de las becas a lo largo del tiempo. Como puede apreciarse, desde el curso académico 2005-2006 tanto la cuantía media de las becas como la dotación presupuestaria crecieron de forma sostenida, acompañadas de un más leve crecimiento del número de beneficiarios del sistema. A partir del curso académico 2012-2013, con la puesta en marcha del nuevo modelo de becas, la dotación presupuestaria se reduce de forma drástica, y solo la estabilización en el crecimiento del número de becarios evitó un desplome aún mayor de la cuantía media de las becas: entre el curso 2011-2012 y el curso 2013-2014 se perdieron 704 euros por beca concedida.

\section{La composición social del estudiantado universitario}

Lo cierto es que, como se apuntaba algo más arriba, alrededor de uno de cada cuatro estudiantes universitarios recibe una beca de la AGE. Ya se decía que valorar la tasa de cobertura exige de algo más que la mera comparación internacional, pues está íntimamente ligada a las distintas características del sistema universitario en su conjunto.

De entre todas ellas, quizás la más importante sea la composición social del estudiantado matriculado en la Universidad, pues las becas se reparten atendiendo criterios de necesidad económica. En función de lo restrictivos o flexibles que sean tales criterios la tasa de cobertura variará notablemente. 
Ahora bien, una vez establecido el umbral por debajo del cual se considera pertinente un apoyo económico, es la composición social del estudiantado universitario la que determina cuántos estudiantes son finalmente receptores de la ayuda. Obsérvese que, si los estudiantes por debajo del nivel de renta fijado tendiesen a no ir a la Universidad, el sistema de becas repartirá menos ayudas simplemente porque hay menos estudiantes que la requieren. Y si, a fin de incrementar la tasa de cobertura pero ante la imposibilidad de que más estudiantes de clases medias-bajas y bajas opten por cursar estudios universitarios, se decidiese flexibilizar los criterios económicos que dan acceso a las ayudas, el sistema terminaría por repartir el presupuesto de becas entre clases sociales cuyas decisiones educativas y rendimiento académico no se ven tan afectados por la mayor disponibilidad de recursos económicos a que da lugar la beca. Examinemos el caso de España.

El sistema de acceso y reparto de las ayudas en nuestro país se estructura a través del establecimiento de distintos umbrales de renta, cuyo número y cuantía se han modificado con la sucesión legislativa descrita en el epígrafe anterior. Los distintos umbrales fijan las condiciones económicas que dan acceso a las diferentes partidas de que se componga la ayuda (o sencillamente la cuantía final de la misma), mientras que el último umbral puede considerarse expresivo de la capacidad económica de un hogar por debajo de la cual se considera necesario apoyar económicamente al estudiante. El cálculo de la renta del hogar tiene en consideración las retribuciones salariales de los miembros del hogar y el valor catastral de sus propiedades excluida la vivienda habitual, estableciendo umbrales diferentes en función del número de miembros que compongan el hogar. La más reciente modificación de los umbrales de renta fue realizada a través del RD 609/2013, fijando su número en tres (Tabla 2).

\begin{tabular}{|l|l|l|l|}
\hline & Umbral $\mathbf{1}$ & Umbral $\mathbf{2}$ & Umbral $\mathbf{3}$ \\
\hline Familias de $\mathbf{1}$ miembro & $3.771 €$ & $13.236 €$ & $14.112 €$ \\
\hline Familias de $\mathbf{2}$ miembros & $7.278 €$ & $22.594 €$ & $24.089 €$ \\
\hline Familias de $\mathbf{3}$ miembros & $10.606 €$ & $30.668 €$ & $32.697 €$ \\
\hline Familias de $\mathbf{4}$ miembros & $13.909 €$ & $36.421 €$ & $38.831 €$ \\
\hline Familias de $\mathbf{5}$ miembros & $17.206 €$ & $40.708 €$ & $43.402 €$ \\
\hline Familias de $\mathbf{6}$ miembros & $20.430 €$ & $43.945 €$ & $46.853 €$ \\
\hline Familias de $\mathbf{7}$ miembros & $23.580 €$ & $47.146 €$ & $50.267 €$ \\
\hline Familias de 8 miembros & $26.660 €$ & $50.333 €$ & $53.665 €$ \\
\hline
\end{tabular}

Tabla 5. Umbrales de renta para la distribución de las becas generales universitarias. Fuente: MECD. 
En el mismo RD 609/2013 quedaron fijadas las distintas cuantías de que se compondrá finalmente la beca, estableciéndose las siguientes modalidades:

- Cuantía fija ligada a la renta: $1500 €$.

- Cuantía fija ligada a la residencia: un máximo de $1500 €$ en caso de acreditar la necesidad de residir fuera del domicilio familiar por motivo de los estudios universitarios matriculados.

- Cuantía variable, con un importe mínimo de $60 €$.

- Beca matrícula.

En función del umbral de renta en que se encuadre al estudiante, este optará a unas u otras de ellas. Para aquellos solicitantes con una renta inferior al primer umbral, las cuatro modalidades estarán disponibles. Si su renta se sitúa entre los umbrales 1 y 2, optará solo a las cuantías b), c) y d). Y en el caso de no superar el tercer umbral, solo obtendrá la beca matrícula.

Se observa por tanto la importancia de la composición social del estudiantado universitario, en tanto que determina tanto la cobertura del sistema como el presupuesto final dedicado a sufragarlo. Y sobre las diferencias en el acceso a la Universidad por clase social en España distintos estudios coindicen en la misma conclusión: las clases sociales medias-bajas y bajas están infrarrepresentadas dentro del estudiantado universitario.

El ya mencionado estudio de Ruesga y Heredero (2010) apunta en esa dirección. Según ambos autores, el nivel medio de recursos económicos anuales de los hogares de los estudiantes que cursan estudios universitarios es un $28 \%$ superior que el nivel económico medio de las familias españolas. Téngase en cuenta que en dicha comparación se contrastan las familias de los universitarios con el conjunto de familias españolas, esto es, el primer conjunto está incluido en el segundo. De compararse las familias con hijos universitarios con las familias sin hijos universitarios, esa diferencia del $28 \%$ sería aún mayor. Aún sin eso, los hallazgos les valen para afirmar que:

Se observa una clara segmentación en el acceso a la Universidad española, de carácter regresivo, primando a los estudiantes con origen familiar más acomodado económicamente, que se acentúa en el caso de las Universidades Privadas (Ruesga y Heredero, 2010, 25).

Conclusiones similares obtienen al comparar el nivel de estudios de los padres universitarios con el característico de la población general. En el caso de las madres de estudiantes universitarios, el 30,5\% ha obtenido un título universitario en el pasado, mientras que entre la población general femenina ese porcentaje asciende al 9,5\%. Igual ocurre con los padres de 
estudiantes universitarios, quienes han finalizado estudios universitarios en el $36,7 \%$ de los casos cuando en la población general masculina han hecho lo propio el 13,5\%. El sesgo en el acceso a la Universidad parece claro tanto en lo que toca al capital económico como al capital cultural del hogar del estudiante.

El estudio de Elias y Pérez (2014), con datos de las Universidades de Barcelona (UB) y Autónoma de Barcelona (UAB), muestra resultados muy similares. Analizan las decisiones sobre el acceso a la Universidad y la titulación finalmente seleccionada en función del origen social, mostrando la preferencia de las clases trabajadoras por carreras de menor duración (las antiguas Diplomaturas) y sus estrategias de compatibilización del tiempo de estudio con la participación en el mercado de trabajo. Encuentran un descenso del $9 \%$ en el número de estudiantes con padres de perfiles formativos bajos tras la implantación del Plan Bolonia que relacionan con ambas cuestiones, pues la adaptación al Espacio Europeo de Educación Superior (EEES) alargó la duración mínima de los estudios universitarios a cuatro años y pasó a exigir una mayor presencia física del estudiante en el aula. La investigación de De Pablos y Gil Izquierdo (2008) arroja de nuevo resultados semejantes. Utilizando la proporción que los precios universitarios representan sobre la renta del hogar, muestran el efecto que las tasas tienen sobre la demanda de educación universitaria. Evidencian cómo, ante una estructura de precios universitarios no dependiente de la renta, menores niveles de poder adquisitivo en el hogar tienen un impacto muy negativo sobre el deseo de acceder a la Universidad.

Por evitar extenderme demasiado, basta apuntar que investigaciones en los niveles previos a la universidad muestran idénticos sesgos en función del origen social en la construcción de la expectativa formativa, esto es, el nivel máximo de estudios que el estudiante espera completar (Elias \& Pérez, 2017). Parece lógico que, si los estudiantes durante la escolarización obligatoria tienden a imaginar su futuro postobligatorio de forma diferencial en función del capital económico y cultural de sus hogares, la composición final del estudiantado universitario se vea notablemente afectada. Y en último término, que se consoliden así barreras que dificulten los procesos de movilidad social ascendente de las clases trabajadoras. Conforme a los estudios de Martínez Celorrio (2013), la reproducción social de las clases altas en España se ve favorecida por los niveles de fracaso escolar (que afecta más a las clases bajas y medias-bajas), un modelo de financiación universitaria no dependiente de la renta y una política de becas modesta comparada con nuestro entorno.

A la luz de estos resultados, la tasa de cobertura del $27 \%$ en el curso 2014-2015, que en la comparación internacional se mostraba muy baja, debe entenderse como el resultado de una notable infrarrepresentación de 
las clases populares en la Universidad y no el efecto ni de un reducido presupuesto para becas (aunque sea, en efecto, un presupuesto reducido) ni de unos umbrales de renta demasiado restrictivos. Por lo primero, recuérdese que todos los estudiantes que satisfacen los criterios económicos y de rendimiento obtienen la ayuda al ser esta considerada un derecho, definiéndose el presupuesto finalmente ejecutado en función de las solicitudes beneficiarias (al menos en las cuantías fijas) y no al revés. En cuanto a los umbrales de renta, y tomando como referencia un hogar de cuatro miembros (según datos del Instituto Nacional de Estadística la mayor proporción de españoles vive en este tipo de hogares con un $28,2 \%$ ), todos los estudiantes universitarios con una renta familiar anual menor a $38.831 €$ tienen garantizado el derecho a la ayuda una vez cumplidas las exigencias de rendimiento. Y de acuerdo con los datos de la ECV publicados por el Instituto Nacional de Estadística, en el año 2013 la renta media nacional de un hogar de 4 miembros fue de $35.752 €$ (Tabla 3 ).

\begin{tabular}{|l|l|l|}
\hline & Umbral 3 & $\begin{array}{c}\text { Renta media } \\
\text { nacional }\end{array}$ \\
\hline Hogar de 1 miembro & $14.112 €$ & $15.187 €$ \\
\hline Hogar de 2 miembros & $24.089 €$ & $26.058 €$ \\
\hline Hogar de 3 miembros & $32.697 €$ & $31.104 €$ \\
\hline Hogar de 4 miembros & $38.831 €$ & $35.752 €$ \\
\hline
\end{tabular}

Tabla 6. Comparación de los umbrales 3 de renta y la renta media nacional por tamaño de hogar. Fuente: Elaboración propia a partir de RD 609/2013 y ECV (Base 2013).

Según datos de Hernández Armenteros y Pérez Díaz (2014), el 65,6\% de los hogares españoles con hijos en edad universitaria presentaron un nivel de renta inferior a los $38.831 €$ que dan acceso a las ayudas. Luego, no parece que el límite sea demasiado restrictivo y penalice a las clases mediasbajas y bajas, pues es incluso superior al nivel de renta característico del hogar medio español. El Gráfico 3 refuerza esa tesis, observándose la notable evolución que se produjo entre los años 2001 y 2008 en el umbral de acceso a las ayudas al estudio para un hogar de cuatro miembros, llegando a casi a duplicar ese límite acreditativo de la necesidad de apoyo económico. Ese impulso sirvió para que el umbral de acceso al sistema de ayudas al estudio superara la renta media de los hogares españoles. El inicio de la crisis económica y la política de restricción presupuestaria han mantenido estable ese último umbral en los actuales $38.831 €$. Los datos apuntan, por tanto, hacia una progresiva flexibilización de los requisitos de renta del sistema de becas, llevando la distribución de las ayudas hacia clases sociales con una disponibilidad de renta por encima de la media nacional. 


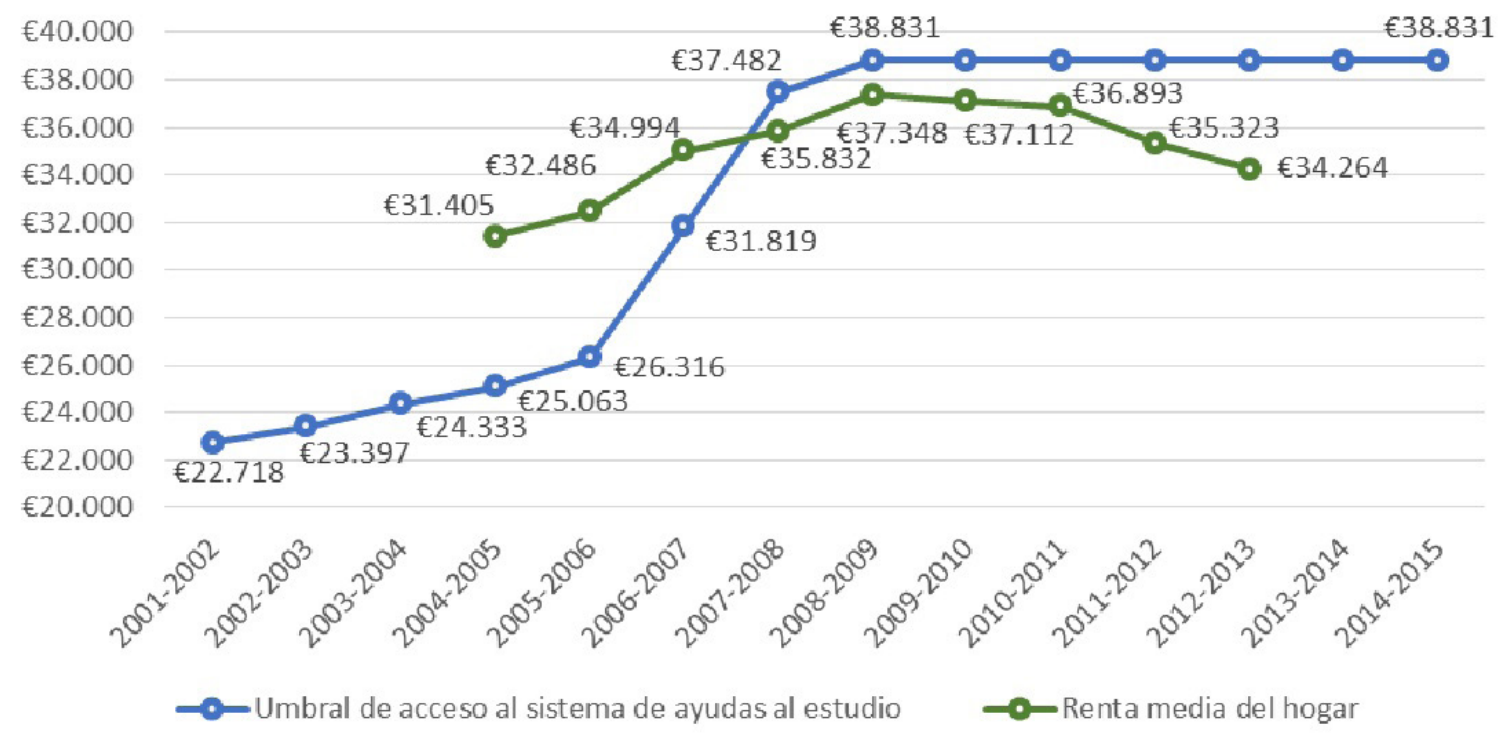

Gráfico 3. Último umbral establecido para el acceso a las ayudas al estudio de la AGE y renta media para hogares de cuatro miembros. Fuente: Elaboración propia a partir de las convocatorias de becas de los años 2001-2014 y la ECV (Base 2004).

Más allá del acceso a la ayuda, la distribución de los solicitantes adjudicatarios de beca en función del tramo de renta en que fueron ubicados es también muy reveladora. Como puede observarse en el Gráfico 4, el tramo de renta con una mayor proporción de beneficiarios es el segundo, acumulando un 52,7\% del total de estudiantes con beca. Nótese, además, que el tercer umbral acumula el 2,2\% de los adjudicatarios, quienes accederán únicamente a la exención de tasas. Pero, si se recuerda la Tabla 1, el 19,3\% de los beneficiarios del sistema habían recibido únicamente la beca matrícula. La diferencia entre ambos porcentajes se nutre de todos aquellos estudiantes pertenecientes al umbral 2 de renta que no satisficieron los criterios académicos para optar a la cuantía variable pero sí los necesarios para la beca matrícula.

En total, y dada la escasa diferencia cuantitativa entre los umbrales segundo y tercero, puede decirse que el $45,1 \%$ de estudiantes en el primer umbral es complementado por un 54,9\% de estudiantes becarios por encima de dicho límite. Es decir, que más de la mitad del estudiantado universitario becado pertenece a un hogar con una renta que, de componerse de cuatro miembros, oscila entre $13.909 €$ y $38.831 €$. Y si bien para el límite inferior parece plenamente justificada la necesidad de apoyo económico, el límite superior puede ser considerado como generoso dada la distribución de rentas de nuestro país. 


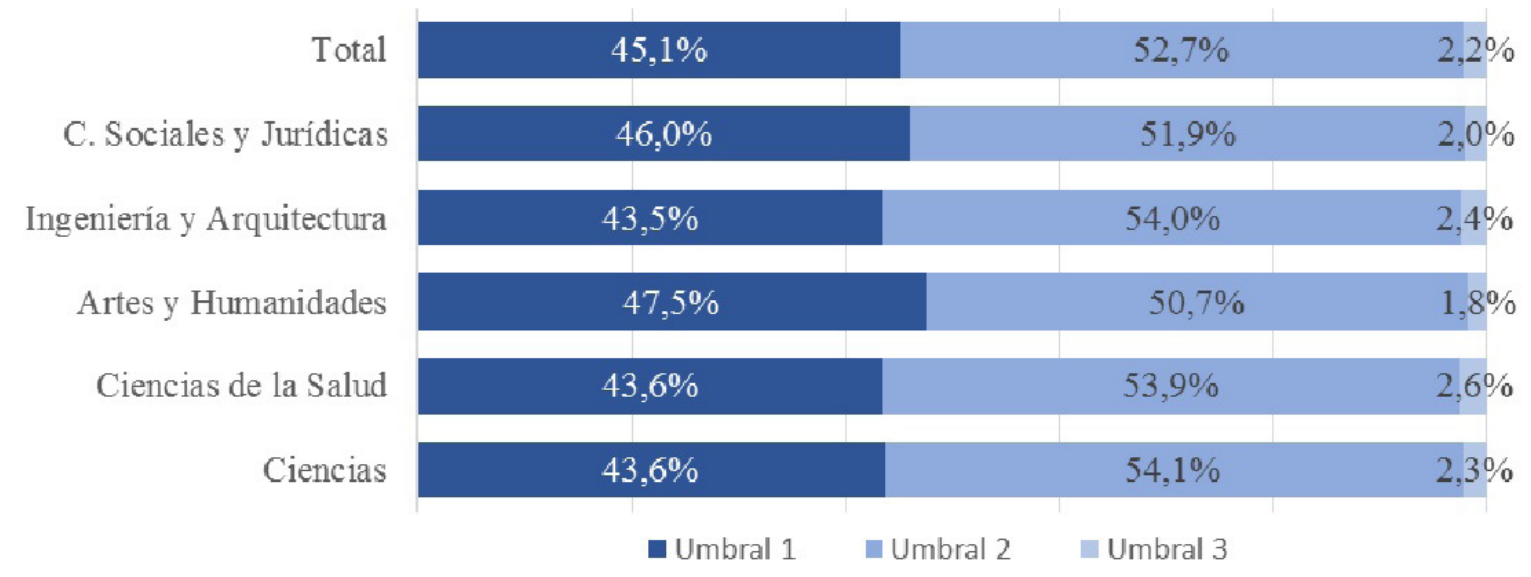

Gráfico 4. Distribución de los beneficiarios del sistema de becas por umbral de renta. Fuente: MECD, 2016.

Pareciera, en conclusión, que el progresivo, aunque lento aumento de las tasas de cobertura en España ha tenido más que ver con el establecimiento de umbrales de renta que trascienden la habitual disponibilidad económica de las clases trabajadoras que con un aumento de la presencia de estas últimas en la Universidad. Y como resultado, que una parte significativa del presupuesto destinado a becas se dirija a un estudiantado, el perteneciente a hogares con niveles de renta alrededor de la media nacional, cuyas decisiones educativas y rendimiento no se ven tan condicionados por la mayor disponibilidad de recursos a que da lugar la beca.

Y en la misma línea, la cuantía media de las becas podría considerarse suficiente para estudiantes de clases medias-bajas y medias para quienes un pequeño apoyo económico mensual que cubra ciertos costes indirectos es capaz de desencadenar los efectos positivos descritos anteriormente. Pero en ningún caso podrán esas cuantías, de forma generalizada, disuadir a un potencial estudiante de clase baja de insertarse profesionalmente ante una situación de acusada necesidad económica. Recuérdese que la cantidad media efectivamente ingresada por aquellos beneficiarios del sistema de becas que obtienen una cantidad dineraria una vez eximidos del pago de las tasas es de $2.088 €$ al año, es decir, $174 €$ al mes. Esa cuantía, una cuarta parte del Salario Mínimo Interprofesional neto, es claramente insuficiente a la hora de cubrir los costes de oportunidad asociados a la matriculación en estudios universitarios, es decir, las retribuciones salariales que el estudiante deja de ganar en el mercado de trabajo. 


\section{Un modelo alternativo de financiación universitaria y de ayudas al estudio}

Tras todo lo dicho, no parece descabellado plantear la necesidad de una reforma del sistema de becas dirigida a incrementar la probabilidad de acceso de las clases más bajas a la Universidad. Dicha reforma no puede tener lugar, sin embargo, ajena a la estructura de financiación del sistema universitario en su conjunto, cuya necesaria redefinición es también un punto común en la literatura académica (De Pablos \& Gil Izquierdo, 2008; Hernández Armenteros \& Pérez García, 2011; Pérez Díaz \& Hernández Armenteros, 2013; Gil Izquierdo \& Carta, 2014; Hernández Armenteros \& Pérez García, 2014).

Las universidades españolas disponen, fundamentalmente, de tres mecanismos de financiación para el mantenimiento de su actividad. El primero de ellos lo constituyen los precios por crédito matriculado asumidos por los estudiantes o cubiertos a través de las becas matrícula. La segunda vía de financiación deviene de las subvenciones públicas, las cuáles cubren alrededor de tres cuartas partes del coste real de los estudios. Y la tercera incluye todos aquellos ingresos provenientes de las distintas actividades de formación, investigación y transferencia llevadas a cabo por las Universidades, así como aquellos otros derivados de estrategias de fundraising que en nuestro país desempeñan un papel residual (Pérez Esparrells, 2013).

En relación a los precios de matrícula de la Universidad española, el Real Decreto-ley 14/2012 impulsó una reforma de gran calado que ha provocado notables incrementos en la proporción de la financiación universitaria asumida por los estudiantes y sus familias. Con el nuevo modelo, las Comunidades Autónomas tienen libertad para fijar el precio final del crédito entre un $15 \%$ y un $25 \%$ del coste real de los estudios, lo que ha provocado una fuerte disparidad en los precios públicos universitarios entre las distintas Comunidades Autónomas (Escardíbul et al., 2014; Hernández Armenteros \& Pérez Díaz, 2015). Disparidad que, por otra parte, ya había comenzado a agudizarse desde la adaptación al EEES y que afecta a la igualdad de acceso entre estudiantes de distintas regiones a lo largo del país (Escardíbul et al., 2014). Sin embargo, ese significativo aumento de los precios de matrícula no ha evitado que entre los años 2008 y 2013 el volumen de ingresos por operaciones no financieras de las universidades españolas se redujera en un $11,38 \%$, lo que pone de manifiesto el notable descenso de la subvención pública del sistema universitario (Hernández Armenteros \& Pérez García, 2015). Pese a todo, la mayor parte del coste real de los estudios universitarios sigue siendo asumida por la Administración, rasgo característico del modelo de financiación universitario típico en Europa Continental donde es habitual una subvención pública superior al 75\% del coste total (Pérez Díaz \& Hernández Armenteros, 2013). 
El estudiantado universitario español queda así dividido en dos grupos: aquellos estudiantes que denominamos becarios y cuyo coste de estudios es asumido íntegramente por el Estado, y aquellos otros no beneficiarios del sistema de becas para los que la subvención cubre tres cuartas partes del coste. El sistema, así definido, termina por ser regresivo en su conjunto (Gil Izquierdo y Carta, 2014), pues la estructura de precios públicos no depende de la capacidad económica del hogar del estudiante y la renta solo interviene a la hora de determinar si se recibe una ayuda al estudio, en cuyo caso se abona un precio de cero euros. Esta disfunción, muy relevante en lo que toca al acceso de las clases bajas a la Universidad, provoca la injerencia del sistema de becas en lo que debiera ser una política de precios universitarios dependiente de la renta. Al establecerse las tasas de matrícula de forma ajena al poder adquisitivo de los hogares, el sistema de becas trata de corregir, sin lograrlo, la regresividad del sistema a través de las becas matrícula.

Distintas investigaciones han insistido en la necesidad de una reforma del modelo de financiación de la Universidad española que permita no solo la suficiencia de recursos con que desempeñar su actividad, sino que también incentive la participación de las clases bajas en los niveles universitarios. De Pablos y Gil Izquierdo (2008) muestran el potencial efecto de un conjunto de reformas relativas a la financiación universitaria y el sistema de becas sobre el deseo de acudir a la Universidad de la población española de entre 17 y 25 años. Para ello, modelizan la demanda de estudios universitarios a partir de datos de la ECV de 2004, aplicando después distintas microsimulaciones que permiten determinar el impacto en términos de probabilidad de acceso a la Universidad de cuatro situaciones hipotéticas: la elevación de las tasas universitarias en un $20 \%$ (que, por sí misma, desincentiva el acceso a la Universidad), la elevación de la subvención pública de cada estudiante en un idéntico $20 \%$, el incremento de la cobertura del sistema de becas y el aumento de la cuantía de las ayudas al estudio (medidas que, por sí mismas, incentivan el acceso a la Universidad). Aplicadas todas de forma conjunta, y dada la función de demanda estimada, se observaría un incremento del $8 \%$ en el número de nuevos estudiantes universitarios, aumento que favorecería especialmente a aquellos estudiantes de hogares más desfavorecidos.

Hernández Armenteros y Pérez García (2011, 2015) realizan una propuesta de reforma diferente. Observan que, entre los años 1996 y 2010, la parte privada de la financiación de estudios universitarios, esto es, la asumida por los estudiantes y sus familias, no ha dejado de disminuir en relación a la subvención pública en nuestro país. En opinión de ambos autores, el sistema debería recuperar aquellos niveles de financiación privada, pero haciendo depender los precios públicos de la renta del hogar del estudiante y penalizando las segundas y terceras matrículas también de acuerdo con 
la capacidad económica familiar. La mayor disponibilidad de recursos así generada debiera dirigirse a la financiación de un sistema de ayudas al estudio con una mayor cobertura de becas de gran cuantía para incrementar los niveles de equidad y becas de transporte, residencia y movilidad que favorezcan la eficiencia del sistema. En conjunto, argumentan, la reforma favorecería fundamentalmente a las clases trabajadoras, quienes verían disminuir los precios a abonar por la matriculación y podrían recurrir a un sistema de becas donde las ayudas permiten afrontar de forma solvente los costes indirectos y de oportunidad asociados la continuación de estudios.

También por una estructura no lineal del sistema de precios universitarios abogan Gil Izquierdo y Carta (2014), quienes proponen una fórmula de financiación alternativa describiendo los casos catalán e italiano. El primero de ellos no es, en sí mismo, un modelo de financiación, pues no es competencia autonómica la determinación de la estructura de precios públicos universitaria. De hecho, Cataluña fue la Comunidad Autónoma que registró mayores incrementos en el precio de matrícula en Grado como resultado de la promulgación del ya mencionado Real Decreto-ley 14/2012, con un $80 \%$ de subida tanto en la experimentalidad máxima como en la mínima (Escardíbul et al., 2014). Pasó así a convertirse en la Comunidad Autónoma con los precios de matrícula más elevados del país, haciendo que Medicina en Barcelona costase $1.620 €$ más que en Andalucía o que los estudios de Historia fueran $924 €$ más caros que en Galicia (Hernández Armenteros \& Pérez García, 2014).

\begin{tabular}{|l|l|l|l|l|l|l|l|}
\hline & Tramo 0 & Tramo 1 & Tramo 2 & Tramo 3 & Tramo 4 & Tramo 5 & Tramo 6 \\
\hline Familias de 1 miembro & 14.112 & 14.112 & 16.065 & 17.017 & 17.968 & 21.775 & \\
\hline Familias de 2 miembros & 24.089 & 24.089 & 27.573 & 29.345 & 31.119 & 38.211 \\
\hline Familias de 3 miembros & 32.697 & 32.697 & 36.187 & 37.354 & 38.523 & 43.195 & \multirow{2}{*}{$\begin{array}{l}\text { Rentas } \\
\text { superiores }\end{array}$} \\
\hline Familias de 4 miembros & 38.831 & 38.831 & 42.554 & 43.520 & 44.485 & 48.349 \\
\hline Familias de 5 miembros & 43.402 & 43.402 & 47.450 & 48.416 & 49.381 & 53.245 \\
\hline Familias de 6 miembros & 46.853 & 46.853 & 51.146 & 52.112 & 53.077 & 56.945 \\
\hline Familias de 7 miembros & 50.267 & 50.267 & 54.802 & 55.768 & 56.733 & 60.597 \\
\hline Familias de 8 miembros & 53.665 & 53.665 & 58.441 & 59.407 & 60.372 & 64.236 & \\
\hline
\end{tabular}

Tabla 5. Umbrales de renta en euros para la distribución de las becas generales universitarias. Fuente: MECD.

A la vez que ese significativo aumento de los precios de matrícula, Cataluña puso en marcha las llamadas becas Equitat, a través de las cuáles les es reembolsado a los estudiantes una parte del precio abonado dependiendo de la renta de su hogar, y que logran reducir el precio final entre un $10 \%$ y un 50\% (Escardíbul et al., 2014). Para ello se establecen seis tramos de ren- 
ta que, de nuevo en función del tamaño del hogar y del llamado coeficiente de estructura docente, fijan la cantidad a abonar a cada estudiante. Como puede observarse comparando la Tabla 5 con la Tabla 2, el primer umbral de renta (tramo o) se inicia allí donde lo deja el último umbral de las becas del MECD. De hecho, los tramos o y 1 se fijan en la misma cantidad económica, reservando el primero de ellos para aquellos estudiantes beneficiarios del sistema de becas nacionales y el tramo 1 para aquellos otros que, satisfaciendo los criterios económicos, no cumplieron los de rendimiento y no obtuvieron la ayuda.

Las becas Equitat funcionan por tanto como un elemento corrector que introduce un criterio de renta en la estructura de precios característica del modelo español, pero constituyen más una solución parcial producto de la actual distribución competencial entre Estado y CCAA que una auténtica alternativa eficiente que aplicar al conjunto del país. Téngase en cuenta que el estudiante debe solicitar la beca Equitat, lo que introduce un procedimiento que solo llevaron a cabo en el curso 2012-2013 el 13,14\% de los estudiantes matriculados (Gil Izquierdo y Carta, 2014). A pesar de ello, son una clara muestra de la voluntad de incorporar al modelo de financiación universitaria español criterios de renta que promuevan el acceso de clases más desfavorecidas a la Universidad.

El modelo italiano sí es, en cambio, un auténtico sistema nacional de precios públicos universitarios construido a partir de la capacidad económica de los estudiantes. Para una descripción más detallada del mismo puede recurrirse a Gil Izquierdo y Carta (2014), pero el sistema funciona básicamente a través del denominado Indicador de la Situación Económica Equivalente (ISEE), determinado anualmente y calculado a través de las rentas del hogar y el $20 \%$ de su patrimonio. La información necesaria para su elaboración es proporcionada en la Declaración Sustitutiva Única, y una vez establecido es empleado tanto en la determinación de los precios universitarios como en otro tipo de prestaciones sociales. El ISEE permite determinar el tramo de renta al que pertenece el hogar del estudiante, y en función de este se calcula la contribución estudiantil que termina configurando el precio a abonar por la matriculación; cálculo para el que las universidades italianas gozan de una notable autonomía.

Se establece así un modelo de financiación en función de la renta a priori y no a posteriori como el caso catalán. Sistema que, manteniendo lo demás constante, contribuye notablemente a la equidad en el acceso a la Universidad y que evita la necesidad de hacer intervenir el sistema de becas en la fijación de los precios de matrícula, esto es, en la corrección de la distinta capacidad de las familias para hacer frente a los costes directos asociados a la matriculación en estudios universitarios. El sistema de ayudas al estudio queda así reservado para hacer frente a aquellas situaciones en que un estu- 
diante no puede asumir los costes indirectos y de oportunidad, misión para la cual la dotación media de le beca debe superar notablemente el estándar actual. No obstante, la última reforma del sistema de becas no apunta en esa dirección pues, como se observó en el Gráfico 2, la cuantía media de las ayudas al estudio cayó $704 €$ por beneficiario entre los años 2011 y 2013, y sustituyó las denominadas becas salario por la cuantía fija ligada a la renta a la que optan los estudiantes en el primer umbral.

Las becas salario nacieron con la intención precisamente de favorecer la entrada de los grupos sociales más desfavorecidos a los niveles universitarios en el contexto generado por la adaptación al EEES. El RD 1721/2007 contemplaba un complemento compensatorio en las becas de la AGE, que bajo el nuevo marco del RD 922/2009 y a partir del curso 2009/2010 pasó a denominarse beca salario. La dotación inicial de las ayudas se estableció en $2.816 €$, pero a fin de convertirse en una auténtica renta de sustitución se planeó incrementar la cuantía de las ayudas hasta los 6.500-7.000 € anuales y redefinir los umbrales de renta para el acceso a las mismas a fin de aumentar su cobertura (Berlanga et al., 2013). Sin embargo, antes de ser sustituidas por la beca fija ligada a la renta las becas salario habían alcanzado una cuantía de solo $3.500 €$. En términos de cobertura, y para el curso $2010 / 2011$, la beca salario fue concedida a 32.850 estudiantes, lo que supuso el $8,14 \%$ del total de estudiantes adjudicatarios. Y en cuanto al presupuesto dedicado a sufragarlas, las becas supusieron un desembolso de 114.975.000 $€$, lo que sobre el total ejecutado en becas en el curso académico 2010/2011 representó el 14,25\%. No parece que, ni siquiera tras la decidida apuesta por las becas salario, las ayudas de mayor cuantía supusieran una proporción mayoritaria ni del número de becas finalmente concedido ni del presupuesto destinado a sufragarlas. $Y$ a este respecto solo recordar que, como se dijo al comienzo del presente artículo, el sistema de becas tiene precisamente por objeto modificar las decisiones educativas de aquellas clases sociales que, por su menor disponibilidad económica, difícilmente pueden acceder a la Universidad en ausencia de la ayuda. El esfuerzo presupuestario no parece conducirse en esa dirección.

Las nuevas ayudas dirigidas a hogares con rentas muy bajas presentan una dotación muy inferior a las becas salario (1.500 €, un $43 \%$ menor), estando ahora vinculadas además al cumplimiento de más exigentes criterios de rendimiento. Debe decirse que el establecimiento de dichos requisitos de aprovechamiento académico no responde a ningún criterio de eficiencia y sí al deseo de adelgazar el gasto público en el sistema de becas. Téngase en cuenta que, según datos de la CRUE, mientras que un estudiante becario consigue graduarse en un tiempo medio de 5,2 años, el estudiante no becario lo hace en 7,14 años (Hernández Armenteros y Pérez García, 2014). No parece lógico ampararse en la eficiencia al exigir mayores esfuerzos 
académicos precisamente a aquellos estudiantes que utilizan los servicios universitarios durante un menor tiempo. Piénsese que, además, para los hogares que serían adjudicatarios económicamente de la cuantía fija ligada a la renta, la ayuda es condición imprescindible para su permanencia en la Universidad, y su no renovación significa, indefectiblemente, el abandono. La mayor exigencia académica en términos de mantenimiento de la beca se convierte así en una elevación del rendimiento mínimo para la permanencia en la Universidad, aspecto que, antes que cualquier otra cosa, es un grave atentado contra la igualdad de oportunidades.

\section{Conclusión}

A lo largo del artículo se ha pretendido ofrecer una definición clara del sistema de ayudas al estudio, de la propia noción de beca y de la condición de becario, reflejando el potencial de las ayudas a la hora de incentivar tanto las decisiones educativas de los estudiantes de clases menos favorecidas como su rendimiento académico. Dicha evidencia ponía de manifiesto la importancia de una adecuada configuración y dotación del sistema de becas para lograr que la composición social de los distintos niveles postobligatorios sea representativa de aquella que caracteriza a la población general. En tanto que, como se expuso, las clases menos favorecidas se hallan infrarrepresentadas en la Universidad española, no parece que el sistema de becas esté logrando cumplir ese objetivo de forma plena. Sí decíamos, no obstante, que exigir que el sistema de ayudas al estudio, por sí mismo, sea capaz de revertir enteramente esa situación es una aspiración quimérica, pues hay variables muy relevantes sobre las que no actúa.

España ha alcanzado unos niveles de cobertura relativamente bajos, haciendo parte del sistema de becas a uno de cada cuatro universitarios. Se ha argumentado, no obstante, que dicha proporción no es sino el resultado de la infrarrepresentación de las clases populares en la Universidad, cuya persistencia ha obligado a incrementar de forma progresiva los umbrales de renta que dan acceso al sistema por encima de la capacidad adquisitiva media de los hogares españoles. Así se ha logrado aumentar la tasa de cobertura hasta los niveles actuales, abriendo el sistema a clases sociales cuyas decisiones educativas y rendimiento académico se ven menos influenciados por la mayor disponibilidad económica que supone la beca y, a cambio, dejando de financiar de forma suficiente las ayudas dirigidas a las clases sociales más bajas.

Es ese un camino que debe desandarse, pues el sistema de ayudas al estudio no tiene por objetivo el reparto del presupuesto de becas entre el mayor número de estudiantes posible, sino incentivar la matriculación en estudios superiores de aquellos estudiantes susceptibles de ser convencidos por 
medio de la subvención económica dada la menor disponibilidad de renta que caracteriza su hogar.

El sistema de becas debe repensarse. Deben redefinirse los criterios económicos de dan acceso a las ayudas, haciéndolos auténticamente representativos de la capacidad económica en la que la beca puede desempeñar su papel. En el corto plazo eso significa, seguro, una reducción de la tasa de cobertura del sistema. A medio y largo plazo, en cambio, se daría lugar a un modelo de becas de gran cuantía que permitan afrontar de forma solvente los costes indirectos y de oportunidad del estudio, lo que, sin lugar a dudas, incrementará la participación de las clases bajas en la Universidad haciendo de nuevo crecer los niveles de cobertura. No será el sistema de becas capaz de lograr ese objetivo solo.

La reforma del sistema de ayudas al estudio debe formar parte de una estrategia más amplia orientada a afrontar el efecto del origen social en el itinerario formativo de los estudiantes españoles, involucrando acciones y medidas que se inicien durante la educación básica y a las que otros programas, como las ayudas al estudio, den continuidad en los niveles postobligatorios.

A la vez, el modelo de financiación universitaria debe asumir como objetivo propio el incentivo de la participación de las clases más humildes en la Universidad. Para ello debe abandonar el tradicional modelo lineal de precios de matrícula, por regresivo, y diseñar un mecanismo de contribución que tenga en cuenta el nivel de renta de los hogares. De esta forma, aquellos estudiantes que saldrían del sistema de becas se verían beneficiados por la nueva estructura de precios, y quienes disponen de un mayor poder adquisitivo contribuirán a la financiación del sistema universitaria de acuerdo con su capacidad económica. Como resultado desaparecerían las becas matrícula, racionalizando el sistema sin injerencias de las ayudas al estudio en el precio abonado por los estudiantes. En cualquier caso, el incremento del volumen de recursos privados derivados de la reforma por la mayor contribución de los hogares de mayor renta no debiera derivar en una rebaja de la subvención pública destinada a los niveles universitarios, pues en ningún caso está en el ánimo de la propuesta que aquí se plantea el contribuir a la política de restricción presupuestaria de los últimos años en España. Sí significaría, en cambio, una mayor disponibilidad financiera de las universidades españolas con que afrontar su actividad, de la que se beneficiarían en especial las clases más humildes de nuestro país. 


\section{Legislación citada}

LRIP

Ley $24 / 2005$, de 18 de noviembre, de reformas para el impulso a la productividad. Boletín Oficial del Estado, núm. 277, 19 de noviembre de 2005, pp. 37846 a 37868.

Real Decreto 2298/1983, de 28 de julio, por el que se regula el sistema de becas y otras ayudas al estudio de carácter personalizado.[Disposición derogada]. Boletín Oficial del Estado, núm. 205, 27 de agosto de 1983, pp. 23578 a 23580

Real Decreto 1721/2007, de 21 de diciembre, por el que se establece el régimen de las becas y ayudas al estudio personalizadas. Boletín Oficial del Estado, núm. 15, 17 de enero de 2008, pp. 3496 a 3507.

Real Decreto 922/2009, de 29 de mayo, por el que se establecen los umbrales de renta y patrimonio familiar y las cuantías de las becas y ayudas al estudio del Ministerio de Educación para el curso 2009-2010. Boletín Oficial del Estado, núm. 131, 30 de mayo de 2009, pp. 45311 a 45323

Real Decreto 609/2013, de 2 de agosto, por el que se establecen los umbrales de renta y patrimonio familiar y las cuantías de las becas y ayudas al estudio para el curso 2013-2014, y se modifica parcialmente el Real Decreto 1721/2007, de 21 de diciembre, por el que se establece el régimen de las becas y ayudas al estudio personalizadas. Boletín Oficial del Estado, núm. 185, 3 de agosto de 2013, pp. 56700 a 56721.

Real Decreto-ley 14/2012, de 20 de abril, de medidas urgentes de racionalización del gasto público en el ámbito educativo. Boletín Oficial del Estado, núm. 96, 21 de abril de 2012, pp. 30977 a 30984.

Bibliografía

Andersen, P. \& Nordli, M. (2012). "Class and cultural capital. The case of class inequality in educational performance", Euopean Sociological Review, 28(5), pp. 607-621.

Berlanga, V., Figuera, P. \& Pons-Farnals, E. (2013). "Incidencia de la beca salario: Impacto, perfil y rendimiento de los estudiantes", Revista Electrónica de Investigación y Evaluación Educativa, RELIEVE, 19(2), pp. 1-15.

Bodovsкi, K. (2010). "Parental practices and educational achievement: social class, race, and habitus", British Journal of Sociology of Education, 31(2), pp. 139-156. 
Conferencia de Rectores de las Universidades Españolas, CRUE (2016). La Universidad Española en cifras, 2014/2015. Madrid: CRUE.

Elias Andreu, M. \& Daza Pérez, L. (2014) "Sistema de becas y equidad participativa en la Universidad", Revista de la Asociación de Sociología de la Educación, 7(1), pp. 233-251.

Elias Andreu, M. \& Daza Pérez, L. (2017) “¿Cómo deciden los jóvenes la transición a la educación postobligatoria? Diferencias entre centros públicos y privados-concertados" $R e$ vista de la Asociación de Sociología de la Educación, 10 (1), pp. 5-22.

Escardíbul, J. O., Pérez Esparrells, C., Morales, S. \& De la Torre, E. (2014). "Precios de matrícula en las enseñanzas universitarias por Comunidades Autónomas (1992-2013): un análisis antes y después del Espacio Europeo de Educación Superior", Comunicación presentada al XXI Encuentro de Economía Pública, Girona.

Gil Izquierdo, M \& CARTA, F. (2014). "Una alternativa progresiva a la estructura lineal de precios públicos en la financiación del sistema universitario en España", Estudios de Economía Aplicada, 32(3), pp. 1097-1116.

Hernández, E. \& GonZÁlez, M. J. (2011). "Modelo de ecuación estructural que evalúa las relaciones entre el estatus cultural y económico del estudiante y el logro educativo", Revista Electrónica de Investigación Educativa, 13(2), pp. 188-203.

Hernández Armenteros, J. \& Pérez García, J. A. (2011). "La financiación universitaria española. Nuevas perspectivas en un horizonte de austeridad y compromiso social". En Nuevas perspectivas para la financiación y el gobierno de las Universidades, Fundación Europea Sociedad y Educación (eds). Madrid. pp. 15-56.

Hernández Armenteros, J. \& Pérez García, J. A. (2014). Crisis fiscal, finanzas universitarias y equidad contributiva. Una reformulación del modelo de financiación de los usuarios de servicios educativos universitarios, Cuaderno de Trabajo 7. Madrid: Fundación Europea Sociedad y Educación.

Hernández Armenteros, J. \& Pérez García, J. A. (2015). "La financiación universitaria como instrumento dinamizador de cambio en la universidad pública española", CIAN-Revista de Historia de las Universidades, 18(1), pp. 19-96.

HILLMERT, S \& ЈАСОВ, M. (2003). "Social inequality in higher education: is vocational training a pathway leading to or away from university?" European Sociological Review, 19(3), pp. 319-334.Martínez Celorrio, X. (2013). "Tendencias de movilidad y reproducción social por la educación en España", Revista de la Asociación de Sociología de la Educación, 6(1), pp. 32-48. 
Mediavilla, M. (2010). "Las becas y ayudas al estudio como elemento determinante de la continuidad escolar en el nivel secundario post-obligatorio. Un análisis de sensibilidad a partir de la aplicación del Propensity Score Matching". En: Mancebón-Torrubia, D. Ximénez-de-Embún, J. Gómez-Sancho y G. Giménez Esteban, ed., Investigaciones de Economía de la Educación Volumen 5, pp. 565-582.

Mediavilla, M. (2013). "Heterogeneidad en el impacto de la política de becas en la escolaridad secundaria postobligatoria en España: un análisis por subgrupos poblacionales", Estudios de Economía, 40(1), pp. 97-120.

Mediavilla, M. (2014). "¿Son efectivas las becas en España? Una evaluación de impacto para el nivel secundario post-obligatorio", Revista Iberoamericana de Evaluación Educativa, 7(1), pp. 121-139.

Ministerio de Educación, Cultura y Deporte, MECD. (2016). Datos y cifras del sistema universitario español. Curso 2015-2016. Madrid: Subdirección General de Documentación y Publicaciones.

Mohamed VÁzquez, R. (2015). "Las ayudas al estudio en la enseñanza superior: una visión comparada entre España y la OCDE”, Ars Iuris Salmanticensis, 3, pp. 125-148.

De Pablos Escobar, L. \& Gil Izquierdo, M. (2008). "Análisis de la incidencia de reformas en el sistema de financiación de la educación universitaria en España a partir de un modelo de comportamiento", Hacienda Pública Española, 184(1), pp. 117-152.

Pérez Esparrells, C. \& Morales, S. (2014). "Las becas y ayudas al estudio en la educación no universitaria en España. Diagnóstico desde la perspectiva regional y propuestas de mejora", Revista de Educación, 366, pp. 87-112.

Pérez García, J. A. \& Hernández Armenteros, J. (2013). Instrumentos para una financiación eficaz de las universidades, Cuaderno de Trabajo 5. Madrid: Fundación Europea Sociedad y Educación.

Río Ruiz, M. A. (2014). "Efectos de la conversión en becario y consecuencias de la reforma del sistema de becas entre universitarios de clase obrera", Revista de la Asociación de Sociología de la Educación, 7(2), pp. 468-487.

Río Ruiz, M. A. \& Jiménez Rodrigo, M. J. (2015). "Políticas de transferencias de rentas condicionadas a la educación en España. El programa de becas 6000", Gestión y Política Pública, 24(2), pp. 377-416. 
Ruesga Benito, S. \& Heredero de Pablos, M. I. (2010). Igualdad en el acceso y la estancia en la universidad en España. Madrid: Ministerio de Educación y Ciencia.

SCANDURRA, R. (2013). "La persistente influencia del origen social sobre la desigualdad de resultados. Una aproximación multi-nivel a partir de PISA 2009", Revista de la Asociación de Sociología de Educación, 6(2), pp. 389-407.

Stavin, R. E. (2010). "Can financial incentives enhance educational outcomes? Evidence from international experiments", Educational Research Review, 5, pp. 68-80.

Ustrell, M. (2012). "La vía profesional-superior disminuye la desigualdad social en el acceso al sistema universitario", Revista de la Asociación de Sociología de la Educación, 5(1), pp. 75-90. 